\title{
The Growth Kinetics of Collision Nodal Metastasis from Medullary and Papillary Thyroid Carcinomas: A Case Report
}

\author{
Hao Lia,b Nir Livneh ${ }^{a, c}$ Snjezana Dogan ${ }^{d}$ Ashok R. Shaha ${ }^{a}$ \\ ${ }^{a}$ Head and Neck Surgery, Memorial Sloan Kettering Cancer Center, New York, NY, USA; ' Otorhinolaryngology, \\ Tan Tock Seng Hospital, Singapore, Singapore; 'O Otolaryngology, Head and Neck Surgery, Sheba Medical Center, \\ Ramat Gan, Israel; ${ }^{d}$ Pathology, Memorial Sloan Kettering Cancer Center, New York, NY, USA
}

\section{Keywords}

Growth kinetics · Collision nodal metastasis · Medullary

thyroid carcinoma Papillary thyroid carcinoma

\begin{abstract}
Introduction: The collision of medullary (MTC) and papillary thyroid carcinoma (PTC) in the same cervical lymph node can occur, but its growth kinetics has not been reported. Case Presentation: We report a 27-year-old male patient who had collision nodal metastases from PTC and sporadic MTC in the central compartment. This was treated with total thyroidectomy and central neck dissection. The collision nodal metastasis persisted and presented with a single sonographically enlarging central compartmental lymph node postoperatively. The volume of the collision nodal metastasis increased from 226 to $507 \mathrm{~mm}^{3}$ over the first 8 months, from 507 to 572 $\mathrm{mm}^{3}$ over the next 6 months, and from 572 to $762 \mathrm{~mm}^{3}$ over the next 31 months. The calcitonin and carcinoembryonic antigen (CEA) fluctuated in the first 19 months followed by a steady increase at a doubling time of 1.97 and 8.42 years, respectively. Unstimulated thyroglobulin remained at 0.2 $\mathrm{ng} / \mathrm{mL}$ or lower during the same period while thyrotropin (TSH) was not suppressed. Revision central neck dissection
\end{abstract}

performed 4.5 years later resulted in undetectable serum calcitonin, CEA of $2 \mathrm{ng} / \mathrm{mL}$, and thyroglobulin of $0.1 \mathrm{ng} / \mathrm{mL}$ from a preoperative calcitonin of $212 \mathrm{ng} / \mathrm{L}, \mathrm{CEA}$ of $10 \mathrm{ng} / \mathrm{mL}$, and thyroglobulin of $0.2 \mathrm{ng} / \mathrm{mL}$. Further structural imaging 13.5 months later revealed no evidence of disease. Discussion: The growth kinetics of collision nodal metastasis from PTC and MTC can be similar to conventional PTC and MTC. Furthermore, the growth rate of such collision nodal metastases can be slow. Guided by tumor marker doubling time and regular structural imaging, surgical salvage performed after a period of active surveillance may still result in biochemical and structural remission.

(C) 2020 European Thyroid Association Published by S. Karger AG, Basel

\section{Introduction}

Papillary thyroid carcinoma (PTC) and medullary thyroid carcinoma (MTC) are thyroid neoplasms derived from different cell types. While the former is common,

Hao Li and Nir Livneh contributed equally to the manuscript. Prior Presentation: abstract accepted by the now canceled 2020 Annual Congress of the American Head and Neck Society. 


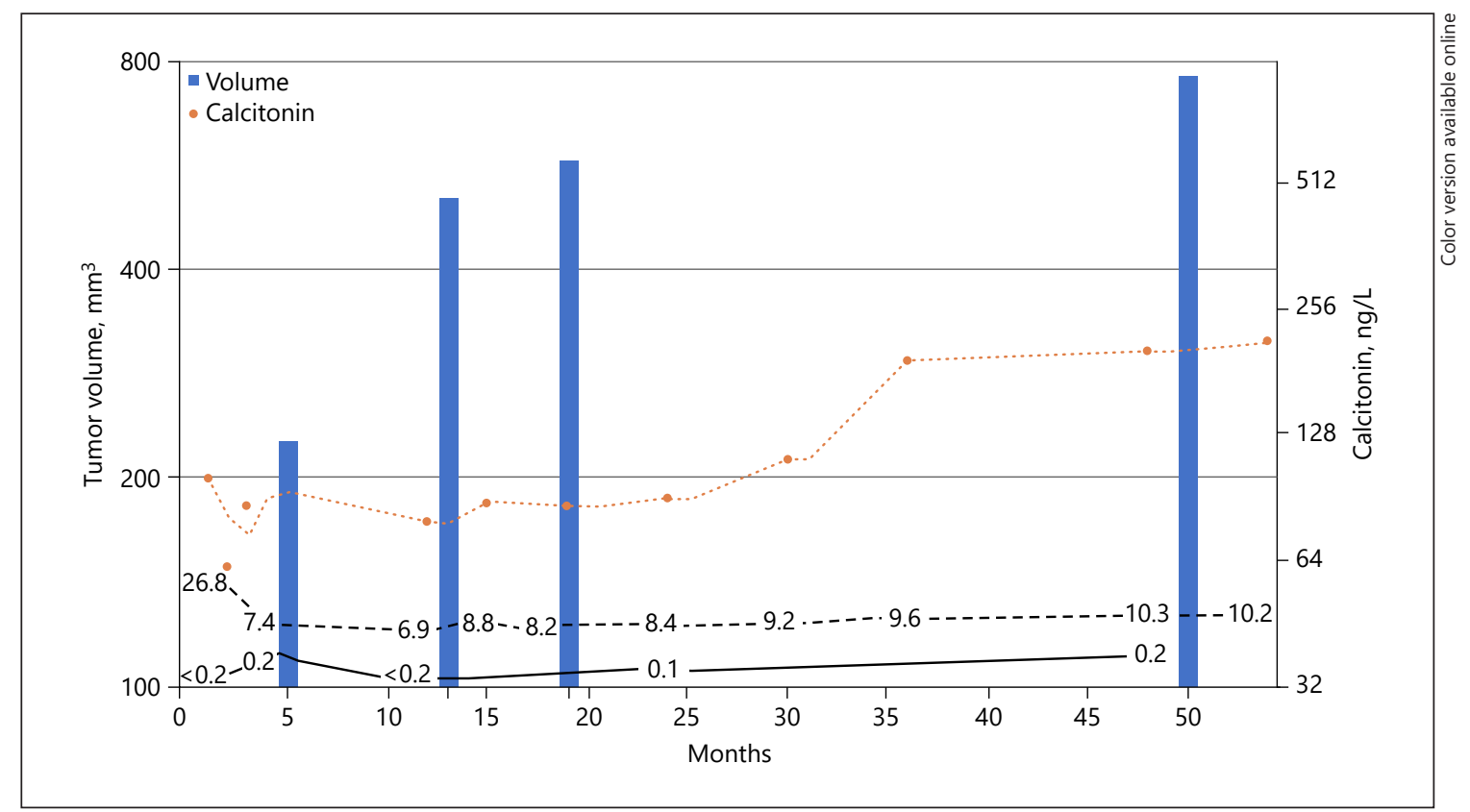

Fig. 1. Growth kinetics of collision nodal metastases from MTC and PTC in a 27-year-old male patient. Solid bars represent the tumor volume. Dots represent the basal serum calcitonin level. Numbers joined by dash are the serum carcinoembryonic antigen levels $(\mathrm{ng} / \mathrm{mL})$. Numbers joined by solid lines are the serum thyroglobulin levels $(\mathrm{ng} / \mathrm{mL})$. The anti-thyroglobulin antibody levels were $<12 \mathrm{IU} / \mathrm{mL}$ at the same time points. TSH was not suppressed. A base 2 logarithmic scale is used for the vertical axes. TSH, thyrotropin; MTC, medullary thyroid carcinoma; PTC, papillary thyroid carcinoma.

the latter is much less frequent $[1,2]$. The synchronous occurrence of both tumors in the same thyroid gland is observed in $14.7 \%$ of thyroidectomies performed for medullary thyroid carcinomas in a series of 82 cases [3]. However, collision metastases of these 2 tumors in the same cervical lymph node have only been sparsely described in the English literature [4-9]. In this report, we describe a patient with collision of MTC and PTC in a nodal metastasis that persisted in the central compartment despite initial surgery. We observed closely the structural and biochemical growth in this metastasis and achieved structural and biochemical remission with a revision central neck dissection 4.5 years later. This report aims to shed light on the growth kinetics of collision nodal metastasis from MTC and PTC in order to understand its pathogenesis and discuss the timing of revision surgery on this condition.

\section{Case Presentation}

The patient was a 27 -year-old male in whom a dentist palpated left thyroid nodules during a routine visit. His thyroid function was normal. Ultrasound showed a $1.6 \mathrm{~cm}$ complex solid nodule in the mid-pole of the left lobe and another $1.9 \mathrm{~cm}$ complex solid nodule in the mid to lower pole of the left lobe. In addition, there were 2 complex solid lymph nodes in left levels III and IV of the neck measuring 1.6 and $4.7 \mathrm{~cm}$, respectively. Fine-needle aspiration showed the left mid-pole thyroid nodule to be PTC, the left mid-pole to lower pole nodule to be MTC and both left lateral neck nodes to be MTC. His past medical history was significant for psoriasis. He did not recall previous exposure to neck radiation. His family history was notable for PTC in the paternal grandfather but no MTC or multiple endocrine neoplasia syndromes. Genetic testing identified no germ line mutation in the RET proto-oncogene.

Laboratory tests revealed a basal calcitonin level of 3,786 ng/L and carcinoembryonic antigen (CEA) level of $286.8 \mathrm{ng} / \mathrm{mL}$. Parathyroid hormone, serum calcium, and 24-hour urinary catecholamines were within the normal range. Computed tomography (CT) of the neck with intravenous contrast showed lymphadenopathy in the left paratracheal region in addition to left levels III and IV nodes. CT chest showed a nonspecific subcentimeter nodule in the right lower lobe. Positron emission tomography-CT showed FDG-avidity in the left thyroid nodule (SUV 3.2) and left supraclavicular metastasis (SUV 4.2).

We treated the patient with total thyroidectomy, left paratracheal nodal dissection, and left levels IIa, III, IV, and V neck dissection. The pathological staging of this patient was $\mathrm{T} 1(\mathrm{~m}) \mathrm{N} 1 \mathrm{aM} 0$ for the PTC and T1(m)N1bM0 for the MTC. Both tumors collided in 2 out of 2 lymph nodes from the paratracheal region. Only MTC 
metastases were found in the lateral neck (see Pathology). The patient did not experience surgical complications postoperatively.

Six weeks later, the basal calcitonin decreased to $100 \mathrm{ng} / \mathrm{L}$, CEA decreased to $26.8 \mathrm{ng} / \mathrm{mL}$, thyroglobulin (TG) was $<0.2 \mathrm{ng} / \mathrm{mL}$, and anti-thyroglobulin antibodies (anti-TG ab) were $<12 \mathrm{IU} / \mathrm{mL}$. No radioiodine was administered and TSH was not suppressed. We continued to survey the patient with calcitonin, CEA, TG, anti-TG $\mathrm{ab}$, free T4 and TSH every 6 months, and ultrasound or neck CT once to twice a year. The trend of his tumor markers is detailed in Table 1 and Figure 1. The first postoperative neck ultrasound performed 5 months later showed a single left central compartment lymph node measuring $9 \times 8 \times 6 \mathrm{~mm}$ medial to the common carotid artery. During monitoring, this lymph node gradually increased to $11 \times 11 \times 8 \mathrm{~mm}$ thirteen months postoperatively, $13 \times$ $12 \times 7 \mathrm{~mm}$ nineteen months postoperatively, and $14 \times 13 \times 8 \mathrm{~mm}$ fifty months postoperatively. CT neck performed during the same period confirmed the lack of additional locoregional disease. CT chest showed the right lower lobe nodule to remain stable over the next 4 years. As the persistently enlarging central compartment lymph node corresponded with rising calcitonin, CEA, and TG, we diagnosed it as residual disease and advised the patient on revision left central neck dissection.

The operation went uneventfully. The left level VI of the neck was dissected with preservation of the recurrent laryngeal nerve. A total of 7 lymph nodes were identified on pathology, 3 out of these contained metastases. All of which showed a collision of both MTC and PTC (Fig. 2h-k). The patient recovered well. The postoperative calcitonin decreased from 212 to $<5 \mathrm{ng} / \mathrm{L}$, and CEA decreased from 10 to $2.3 \mathrm{ng} / \mathrm{mL} 8$ weeks later. Thirteen and half months later, his calcitonin remained $<5 \mathrm{ng} / \mathrm{L}$, CEA was $2 \mathrm{ng} / \mathrm{mL}$, thyroglobulin was $0.1 \mathrm{ng} / \mathrm{mL}$, anti-thyroglobulin antibody was $0.9 \mathrm{IU} / \mathrm{mL}$, and ultrasound neck showed no structural disease.

\section{Pathology}

Immunohistochemistry (IHC) for PTC and MTC including thyroglobulin, TTF-1, PAX8, mCEA, calcitonin, synaptophysin, and chromogranin was performed using the Ventana Benchmark Ultra platform (Ventana Medical Systems Inc., Tucson, AZ, USA) as per manufacturer's protocol. Details on antibodies and conditions are provided in the online suppl. Table; for all online suppl. material, see www.karger.com/doi/10.1159/000511184.

The thyroidectomy specimen revealed multiple foci of MTC and PTC, both ranging from 0.1 to $1.5 \mathrm{~cm}$ in greatest dimension located in the left lobe (Fig. 2). All PTC foci were classical type, encapsulated with at least capsular invasion, while among the MTC foci; only the largest one was encapsulated. There was no vascular invasion identified and no extrathyroidal extension by either of the tumors. The surgical margins were free of cancer and the nonneoplastic thyroid exhibited nodular hyperplasia. The MTC was confirmed by positive IHC for chromogranin, synaptophysin, calcitonin, mCEA, TTF-1, and negative PAX8 and thyroglobulin immunostains. No C-cell hyperplasia was noted in the histologically normal thyroid parenchyma by light microscopy or by IHC (for chromogranin and calcitonin). On the left neck dissection, 5 out of 36 lymph nodes were positive for metastatic MTC, with the largest lymph node, $4.2 \mathrm{~cm}$ in size, completely replaced by tumor but without extranodal extension. In addition, 2 out of 2 left paratracheal lymph nodes were positive for both metastatic MTC and PTC with the MTC deposit being the larger one ( 0.32 vs. 0.1 $\mathrm{cm}$ ). The pathology of the revision central neck dissection 4.5 years

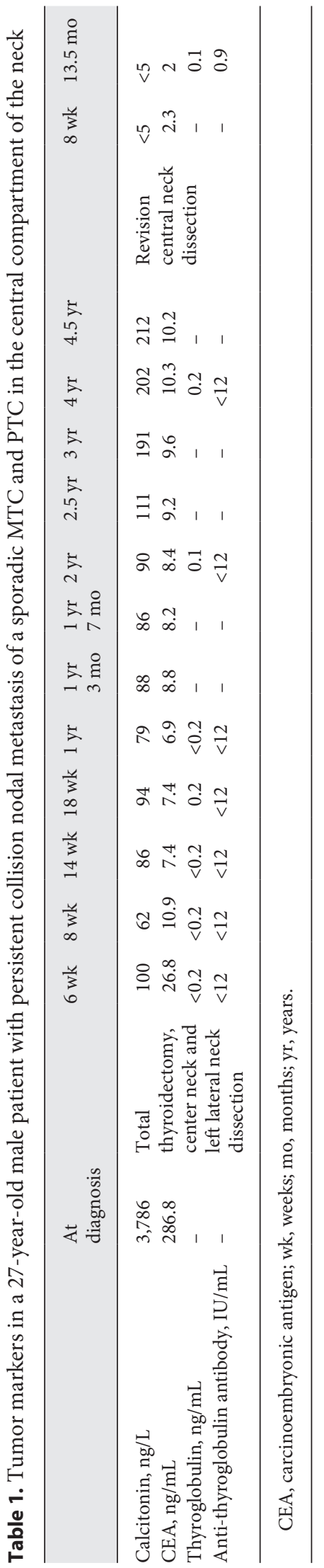

Growth Kinetics of Collision Nodal

Metastasis from MTC and PTC
Eur Thyroid J 2021;10:345-352 DOI: $10.1159 / 000511184$ 

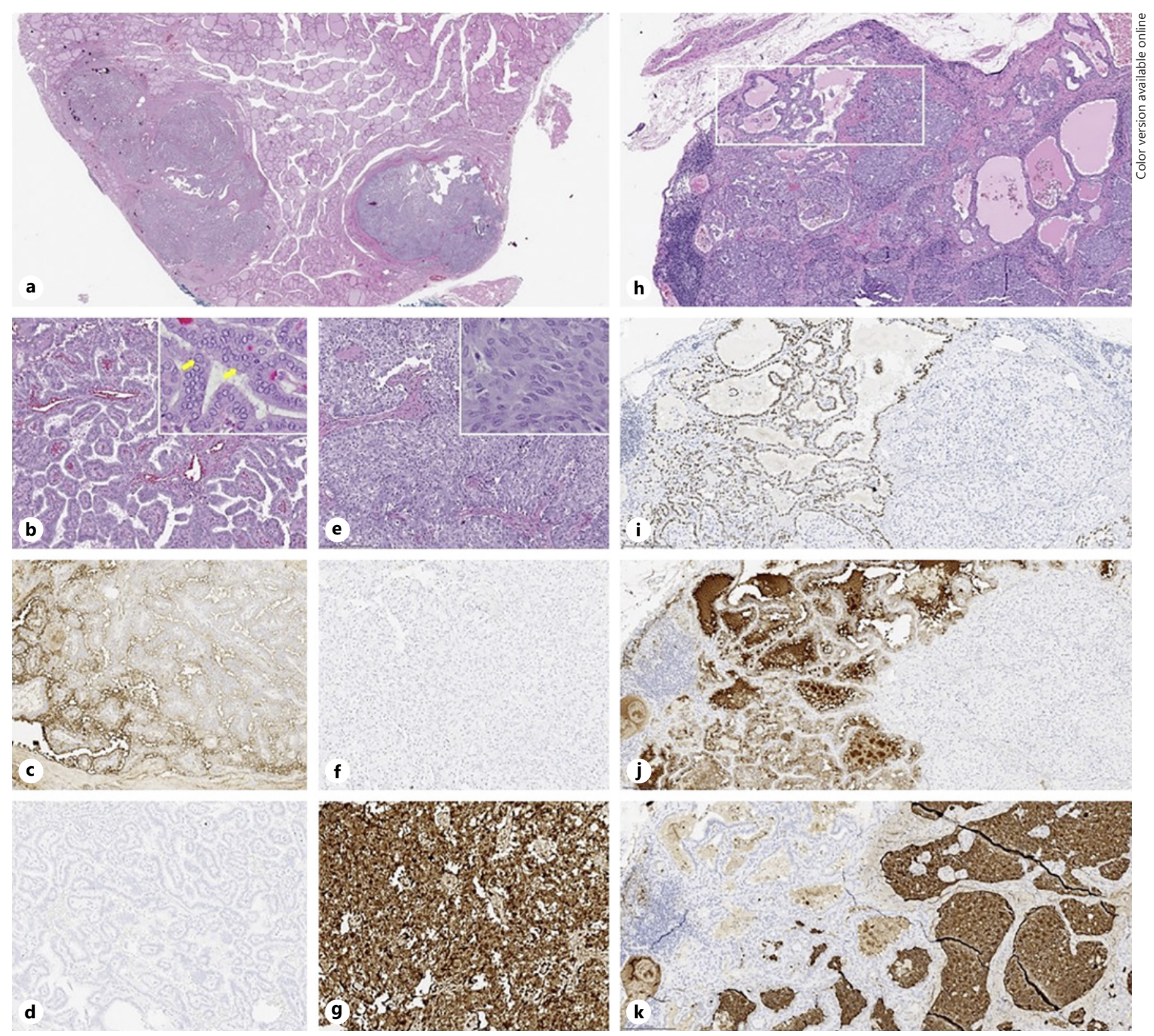

Fig. 2. Pathological features of the collision nodal metastasis from MTC and PTC. a-g Show synchronous primary PTC and MTC. h-k Show collision metastases in paratracheal lymph nodes. Thyroid section shows the primary tumor nodules of the PTC (left) and MTC (right; a, H\&E, low power view). PTC exhibited a classical papillary morphology (b, H\&E, $\times 200$ magnification), enlarged, clear nuclei with scattered intranuclear pseudoinclusions (b, inset, $\times 400$ magnification; yellow arrows). PTC was positive for thyroglobulin (c, $\times 200$ magnification) and negative for chromogranin IHC (d, $\times 200$ magnification). MTC showed nested and solid growth patterns (e, H\&E, $\times 200$ magnification) and was com- prised of polygonal and spindle tumor cells showing nuclei with characteristic "salt and pepper" chromatin (e, inset, $\times 400$ magnification). In contrast to PTC, MTC was negative for thyroglobulin (f, $\times 200$ magnification) and positive for chromogranin IHC (g, $\times 200$ magnification). Metastases from both tumors collided in the same lymph node (h, H\&E, low power view; white framed area is seen in IHC sections at $\times 200$ magnification). PAX8 IHC (i) and thyroglobulin immunostains highlight only the PTC component on the left (j), while chromogranin IHC labels only MTC metastasis on the right (k). IHC, immunohistochemistry; MTC, medullary thyroid carcinoma; PTC, papillary thyroid carcinoma. 


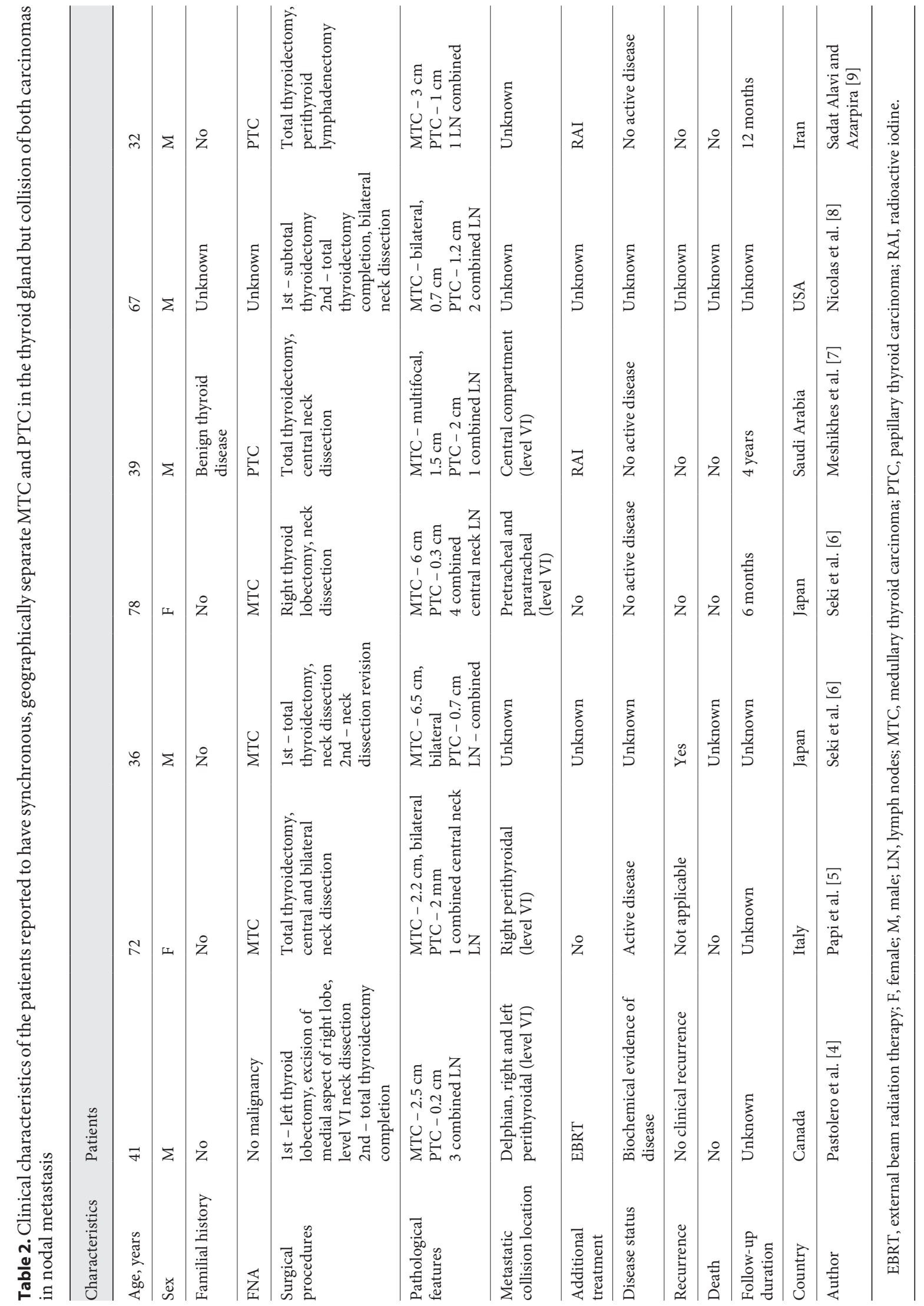


later showed that the 3 lymph nodes harboring both MTC and PTC had similar morphology to those seen in the first surgical specimen. The size of the largest metastatic focus was $1 \mathrm{~cm}$ containing both medullary and papillary carcinomas, but the medullary component predominated (Fig. 2). The size of the largest focus of papillary carcinoma was $5 \mathrm{~mm}$. There was extranodal extension in only the medullary component.

\section{Discussion}

The prevalence of differentiated thyroid carcinomas, most of which are PTC, is estimated to be $11.2 \%$ on autopsy [10] while the number of new patients with MTC in the USA will only be approximately a thousand in 2020 $[1,2]$. The high prevalence of PTC makes its incidental diagnosis in patients with sporadic MTC likely, given that both PTC and sporadic MTC are generally slow growing and affect adults of similar age-groups [11]. These synchronous carcinomas can present as a single tumor with mixed features, a single tumor with 2 distinct components, or as 2 tumors separated by normal thyroid tissue [12-15].

In some of these patients, cervical lymph node metastasis of 1 or both tumors is present. However, we found only 7 patients in the English scientific literature with features resembling the tumor of our patient, that is, synchronous MTC and PTCs, separated by normal thyroid tissue, with a collision metastasis in the same lymph node [4-9]. Among them, 1 article described the recurrence of such collision metastasis [6]. The features of these cases are summarized in Table 2. Five patients had PTC of $1 \mathrm{~cm}$ or less, while 6 patients had MTC of $1 \mathrm{~cm}$ or more. Four patients had collision nodal metastases in level VI, while in the other patients; the specific location was not reported. The patient had recurrence presented with a collision metastasis of MTC and PTC in the right lateral neck 1 year after surgery. None of the patients died during the follow-up but only 3 investigators reported the duration of the follow-up, which lasted between 6 months and 4 years.

The pathogenesis of synchronous MTC and PTC in the same patient is unclear. A shared oncogenic pathway for these tumors of distinct cellular lineage may be possible. RET germ line mutation affecting codon 804 of axon 14 was reported to be associated with both MTC and PTC [5, 16]. Vantyghem and colleagues [3] described higher prevalence of occult PTC in patients who underwent total thyroidectomy for MTC (14.7\%) compared to other reasons for total thyroidectomy (6.8\%). Fugazzola et al. [17] found high frequency of PTC in a family with familial MTC. However, our patient, as with most other described patients (Table 2), had no familial history of thyroid carcinomas nor germline RET mutations. As the majority of MTC and PTC are sporadic, it might be more likely for the concurrence of these 2 malignancies to be coincidental. This is evidenced by Kim and colleague's [18] observation that most patients with synchronous MTC and PTC have papillary thyroid microcarcinoma while the MTC was $>1 \mathrm{~cm}$, and there was no difference in the rate of identifying PTC in patients treated surgically for MTC (19\%), Grave's disease (15\%), and follicular thyroid cancer (19\%).

The initially observational management of the persistent collision nodal metastasis in our patient offered us an opportunity to examine the growth kinetics of this condition. This information can be important in deciding on the timing of revision neck dissection. In a recently operated neck, inflammation tends to obscure the visualization of critical structures, which are the recurrent laryngeal nerve and the parathyroid glands in the central compartment. Delaying revision central neck surgery for 6 months or more in order to improve the preservation of these structures has been advocated by an experienced surgeon [19]. Active surveillance of small, stable recurrent nodal disease in differentiated thyroid carcinoma has also been endorsed by the American Thyroid Association (ATA) as an alternative to upfront revision lymphadenectomy in selected patients [20]. However, the ATA recommended revision lymphadenectomy for persistent nodal disease in MTC if the serum basal calcitonin level does not exceed $1,000 \mathrm{pg} / \mathrm{mL}$, and 5 or fewer lymph nodes were removed at the initial surgery [2]. Over 4.5 years, we observed in our patient that the sonographic volume (estimated by the ellipsoid formula $\pi / 6 \times$ length $\times$ width $\times$ height) of the node with the collision metastasis increased in a nonexponential manner from 226 to $507 \mathrm{~mm}^{3}$ over 8 months, then from 507 to $572 \mathrm{~mm}^{3}$ over the next 6 months, and from 572 to $762 \mathrm{~mm}^{3}$ over the next 31 months. Correspondingly, the longest dimension of the node increased from 9 to 11 to 13 to $14 \mathrm{~mm}$ at the same time points. This collision nodal metastasis appeared to enlarge faster than the typical recurrent or residual nodal metastasis of PTC which showed a median growth rate of $1.4 \mathrm{~mm}$ per year in a study of 83 patients [21], and enlargement of $3 \mathrm{~mm}$ or more in the longest dimension occurred in only $20 \%$ of 166 PTC patients in another study with a median follow-up of 3.5 years [22]. This difference could reflect the growth rate of the MTC component of the collision nodal metastasis, which proved to be dominant in final pathology. 
The biochemical growth kinetics observed through calcitonin and CEA showed that these markers fluctuated in the first 19 months of observation, after which they steadily increased at a doubling time of 1.97 and 8.42 years, respectively (estimated from the data in Table 1 using the ATA calculator [23] beginning 19 months postoperatively when calcitonin and CEA reached a nadir after the initial fluctuation). These doubling times are observed in patients with isolated MTCs. In a meta-analysis of 73 patients with MTC, $16.4 \%$ had a calcitonin doubling time between 0.5 and 2 years, and $48 \%$ had a doubling time of $>2$ years. Thirty-nine out of the 73 patients provided prognostic data on CEA doubling time, and $36 \%$ had a CEA doubling time of $>2$ years [24]. The thyroglobulin doubling time in our patient could not be determined accurately because his TSH was not suppressed. When TSH was suppressed to below $0.1 \mathrm{mIU} / \mathrm{L}$, thyroglobulin doubling time of $<1$ year, 1-3 years, 3 years or longer and a decline in thyroglobulin was observed in $12.4 \%$ (17 out of 137 ), $15.3 \%$ ( 21 out of 137 ), $21.9 \%$ (30 out of 137), and 50.3\% (69 out of 137) patients with PTC who underwent total thyroidectomy [25]. Observing the thyroglobulin doubling time of collision nodal metastasis from PTC and MTC while maintaining TSH at a stable level requires further study.

The timing of the revision lymphadenectomy in this patient is noteworthy for its achievement of a biochemical and structural remission of MTC 4.5 years later. Although we do not advocate delaying surgical salvage for residual nodal disease of MTC, determining the extent of further surgery in this patient was somewhat challenging because the lateral neck and the right lung nodule might also be sites of residual disease. However, regular structural surveillance in the first 2 years pointed to the central neck to be the likely site of residual disease. Persistent enlargement of the lymph node in the central neck in conjunction with a steady rise in tumor markers eventually justified the risks of recurrent laryngeal nerve palsy and hypo- parathyroidism secondary to a revision central neck dissection. This patient illustrates that the growth rate in collision nodal metastases from MTC and PTC can be slow and surgical salvage after a period of active surveillance may be successful. Nonetheless, the oncological outcome of this approach requires prospective studies to determine.

In this report, we describe the structural and biochemical growth kinetics of collision nodal metastasis from MTC and PTC in a young adult patient. The slow growth of the collision nodal metastases in this patient enabled a revision lymphadenectomy performed 4.5 years later to achieve a biochemical and structural remission of both carcinomas. More reports on the clinical behavior and the management of these collision metastases can further enrich our understanding of this rare condition.

\section{Statement of Ethics}

The patient provided consent to the publication of his medical information in an anonymized manner.

\section{Conflict of Interest Statement}

The authors have no conflict of interest to declare in relation to this publication.

\section{Author Contributions}

Hao Li conceptualized this paper, collected the clinical data, and drafted and revised the manuscript before submission. Nir Livneh performed the literature review of similar cases, prepared the majority of Tables 1 and 2 and Figure 1, and drafted and revised the manuscript before submission. Snjezana Dogan prepared the pathological description of the collision nodal metastasis in the main text, created Figure 2, the online suppl. Table, and reviewed the manuscript before submission. Ashok Shaha conceptualized this paper, provided the clinical material, and reviewed the manuscript before submission.

\section{References}

Growth Kinetics of Collision Nodal

Metastasis from MTC and PTC
1 Noone A, Howlader N, Krapcho M, Miller D, Brest A, Yu M, et al. 2017 SEER cancer statistics review, 1975-2015. Bethesda, MD: Natl Cancer Institute. Available from: www.seer. cancer.gov. Accessed 2020 May 24.

2 Wells SA, Asa SL, Dralle H, Elisei R, Evans DB, Gagel RF, et al. Revised American thyroid association guidelines for the management of medullary thyroid carcinoma. Thyroid. 2015; 25(6):567-610.
3 Vantyghem MC, Pigny P, Leteurtre E, Leclerc L, Bauters C, Douillard C, et al. Thyroid carcinomas involving follicular and parafollicular C cells: seventeen cases with characterization of RET oncogenic activation. Thyroid. 2004;14(10):842-7.

4 Pastolero GC, Coire CI, Asa SL. Concurrent medullary and papillary carcinomas of thyroid with lymph node metastases: a collision phenomenon. Am J Surg Pathol. 1996;20(2): 245-50. 
5 Papi G, Corrado S, Pomponi MG, Carapezzi C, Cesinaro A, LiVolsi VA. Concurrent lymph node metastases of medullary and papillary thyroid carcinoma in a case with RET oncogene germline mutation. Endocr Pathol. 2003; 14(3):269-76.

6 Seki T, Kameyama K, Hayashi H, Nagahama M, Masudo K, Fukunari N, et al. Composite metastatic carcinoma in lymph nodes of patients with concurrent medullary and papillary thyroid carcinoma: a report of two cases. Endocr Pathol. 2004;15(1):83-8.

7 Meshikhes AW, Tingura M, Al-Saeed JY. Concurrent papillary and medullary thyroid carcinomas with mixed metastases to lymph nodes. Saudi Med J. 2004;25(3):373-5.

8 Nicolas MM, Neto AG, Luna MA. Concurrent papillary and medullary thyroid carcinoma. Arch Pathol Lab Med. 2005;129(2):2645.

9 Sadat Alavi M, Azarpira N. Medullary and papillary carcinoma of the thyroid gland occurring as a collision tumor with lymph node metastasis: a case report. J Med Case Rep. 2011;5:590.

10 Furuya-Kanamori L, Bell KJ, Clark J, Glasziou P, Doi SA. Prevalence of differentiated thyroid cancer in autopsy studies over six decades: a meta-analysis. J Clin Oncol. 2016; 34(30):3672-9.

11 Randle RW, Balentine CJ, Leverson GE, Havlena JA, Sippel RS, Schneider DF, et al. Trends in the presentation, treatment, and survival of patients with medullary thyroid cancer over the past 30 years. Surgery. 2017;161(1):13746.
12 Jain M, Verma D, Thomas S, Chauhan R. Mixed medullary: papillary carcinoma thyroid: an uncommon variant of thyroid carcinoma. J Lab Physicians. 2014;6(2):133-5.

13 Wong RL, Kazaure HS, Roman SA, Sosa JA. Simultaneous medullary and differentiated thyroid cancer: a population-level analysis of an increasingly common entity. Ann Surg Oncol. 2012;19(8):2635-42.

14 Ryan N, Walkden G, Lazic D, Tierney P. Collision tumors of the thyroid: a case report and review of the literature. Head Neck. 2015; 37(10):E125-9.

15 Lax SF, Beham A, Kronberger-Schönecker D, Langsteger W, Denk H. Coexistence of papillary and medullary carcinoma of the thyroid gland-mixed or collision tumour? Clinicopathological analysis of three cases. Virchows Arch. 1994;424(4):441-7.

16 Brauckhoff M, Gimm O, Hinze R, Ukkat J, Brauckhoff K, Dralle H. Papillary thyroid carcinoma in patients with RET proto-oncogene germline mutation. Thyroid. 2002;12(7):55761.

17 Fugazzola L, Cerutti N, Mannavola D, Ghilardi G, Alberti L, Romoli R, et al. Multigenerational familial medullary thyroid cancer (FMTC): evidence for FMTC phenocopies and association with papillary thyroid cancer. Clin Endocrinol. 2002;56(1):53-63.

18 Kim WG, Gong G, Kim EY, Kim TY, Hong SJ, Kim WB, et al. Concurrent occurrence of medullary thyroid carcinoma and papillary thyroid carcinoma in the same thyroid should be considered as coincidental. Clin Endocrinol. 2010;72(2):256-63.

19 Clayman GL. Technique for completion/reoperative thyroidectomy. In: Chapter 17 of head and neck surgery, vol.2 of master techniques in otolaryngology-head and neck surgery. Philadelphia, PA: Wolters Kluwer Health; 2014.
20 Tufano RP, Clayman G, Heller KS, Inabnet WB, Kebebew E, Shaha A, et al. Management of recurrent/persistent nodal disease in patients with differentiated thyroid cancer: a critical review of the risks and benefits of surgical intervention versus active surveillance. Thyroid. 2015;25(1):15-27.

21 Tomoda C, Sugino K, Matsuzu K, Uruno T, Ohkuwa K, Kitagawa W, et al. Cervical lymph node metastases after thyroidectomy for papillary thyroid carcinoma usually remain stable for years. Thyroid. 2016;26(12):1706-11.

22 Robenshtok E, Fish S, Bach A, Domínguez JM, Shaha A, Tuttle RM. Suspicious cervical lymph nodes detected after thyroidectomy for papillary thyroid cancer usually remain stable over years in properly selected patients. J Clin Endocrinol Metab. 2012;97(8):2706-13.

23 American thyroid association calcitonin and carcinoembryonic antigen (CEA) doubling time calculator. Available from: https://www. thyroid.org/professionals/calculators/thyroid-cancer-carcinoma/ Accessed $2020 \mathrm{Jul}$ 23.

24 Meijer JA, le Cessie S, van den Hout WB, Kievit J, Schoones JW, Romijn JA, et al. Calcitonin and carcinoembryonic antigen doubling times as prognostic factors in medullary thyroid carcinoma: a structured meta-analysis. Clin Endocrinol. 2010;72(4):534-42.

25 Miyauchi A, Kudo T, Miya A, Kobayashi K, Ito Y, Takamura Y, et al. Prognostic impact of serum thyroglobulin doubling-time under thyrotropin suppression in patients with papillary thyroid carcinoma who underwent total thyroidectomy. Thyroid. 2011;21(7):707-16. 